\title{
Dor lombar intensa em gestantes do extremo Sul do Brasil
}

\author{
Severe low back pain among pregnant women in Southern Brazil
}

Vlanice Madruga Duarte ${ }^{1}$

Rodrigo Dalke Meucci ${ }^{2}$

Juraci Almeida Cesar ${ }^{1}$
${ }^{1}$ Programa de PósGraduação em Ciências da Saúde, Universidade Federal do Rio Grande (FURG). R. Gen. Osório 102/4, Centro. 96203-900 Rio Grande RS Brasil. vlanicemadruga@gmail.com

${ }^{2}$ Programa de Pós-

Graduação em Saúde

Pública, FURG. Rio Grande RS Brasil.

\begin{abstract}
The scope off this article is to assess the prevalence and identify associated factors with severe low back pain (LBP) during pregnancy among mothers living in Rio Grande, in the south of Brazil, in 2013. A standardized questionnaire was applied by trained interviewers within 48 hours after delivery. Multivariate analysis was carried out using Poisson regression. The prevalence ratio was the effect measure used. Among the 2,685 pregnant women enrolled in the study, $51.2 \%$ reported $L B P$ and $27.3 \%$ reported severe LBP, which was more likely to occur among adolescents, with overweight/obese individuals, former smokers and those who practiced physical activity during pregnancy. Among those with severe $L B P, 29.2 \%, 37.3 \%$ and $33.3 \%$ reported limitations for daily activities at home, had to seek medical care and had to miss work due to this problem, respectively. The prevalence of severe LBP during pregnancy is high and its impact for daily life and health services is concerning. The management of $L B P$ should be part of routine pre-natal consultation.
\end{abstract}

Key words Low back pain, Prevalence, Pregnancy
Resumo O objetivo deste artigo é medir a prevalência e identificar fatores associados à dor lombar intensa (DL intensa) entre parturientes do município de Rio Grande, RS, Brasil, em 2013. Questionário padronizado aplicado por entrevistadoras previamente treinadas em até 48 horas após $o$ parto. Realizou-se análise multivariável utilizando regressão de Poisson tendo a razão de prevalência como medida de efeito. Dentre as 2685 parturientes incluídas no estudo, 51,2\% referiram dor lombar gestacional e $27,3 \%$ tinham DL intensa, com maior probabilidade de ocorrência entre adolescentes, mulheres com sobrepeso/obesidade, ex-fumantes e naquelas que praticaram atividade física na gestação. Entre as parturientes que tiveram DL intensa, 29,2\%, 37,3\% e 33,3\% tiveram que deixar de fazer as lidas da casa, faltar ao trabalho e procurar um médico devido à $D L$, respectivamente. É elevada a prevalência de dor lombar intensa no período gestacional e preocupante seu impacto sobre as atividades da vida diária e utilização de serviços de saúde. Recomenda-se investigação clínica e manejo da dor lombar durante a gestação nas consultas de rotina do pré-natal.

Palavras-chave Dor lombar, Prevalência, Gestação 


\section{Introdução}

A dor lombar gestacional (DLG) é a principal queixa de desconforto musculoesquelético durante a gestação e está diretamente relacionada à funcionalidade e à qualidade de vida das gestantes ${ }^{1}$. Juntamente com a dor da cintura pélvica, tem impactos negativos à qualidade de vida no período gestacional e puerperal podendo, inclusive, cronificar. Existem relatos dos impactos econômicos decorrentes do absenteísmo causado pela $\mathrm{DLG}^{2}$.

Entre os principais causadores de DLG estão as alterações fisiológicas inerentes ao período gestacional, tais como mudanças dos níveis hormonais de relaxina, estrogênio e progesterona. $\mathrm{O}$ aumento da flexibilidade articular, o alargamento da sínfise púbica e a acentuação da inclinação pélvica resultam em alterações biomecânicas que levam à adoção de posturas compensatórias que sobrecarregam a coluna lombar, predispondo ao surgimento da dor ${ }^{3}$.

A literatura indica que a DLG é um problema universal de prevalência variável conforme a definição do desfecho ${ }^{2,4}$. Estudos de base populacional conduzidos na Noruega, Estados Unidos e Espanha mostram prevalências de 51\%, 68\% e $71 \%$, respectivamente ${ }^{5-7}$. Entretanto, um estudo que mensurou a severidade da dor estimou uma prevalência de DL intensa de $25 \%^{8-10}$. No Brasil, as estimativas da ocorrência deste desfecho são provenientes de estudos com amostras de conveniência e número reduzido de participantes ${ }^{11-13}$, o que evidencia a carência de estimativas sobre este tema no país.

A literatura mostra que trabalho extenuante e história prévia de dor lombar ou de dor na cintura pélvica são importantes preditores da ocorrência da DLG 2 . Além disso, fatores demográficos, socioeconômicos, reprodutivos e comportamentais também estão associados à DLG, a qual é mais frequente entre mulheres de cor da pele preta, que trabalham fora de casa, com nível intermediário de escolaridade, de maior paridade, maior Îndice de Massa Corporal (IMC) e que praticam atividade física irregular- ${ }^{5-7,10,14}$.

Apesar de ser altamente prevalente e de levar à limitação das atividades de vida diária, a DLG, sobretudo a DL intensa, é pouco avaliada em estudos de base populacional de países de média e baixa renda. O presente estudo tem por objetivos: medir a prevalência e identificar os fatores associados à DL intensa entre parturientes de Rio Grande no ano de 2013; e, descrever a proporção de parturientes com DL intensa que deixaram de realizar as lidas da casa, faltar ao trabalho e procurar por um profissional médico.

\section{Metodologia}

Estudo de delineamento transversal realizado em Rio Grande, município localizado no extremo sul do estado do Rio Grande do Sul. Os dados apresentados foram coletados no ano de 2013 e fazem parte de um estudo mais amplo intitulado "Estudo Perinatal de Rio Grande, RS" que busca, a cada triênio, avaliar a qualidade da assistência à gestação e ao parto oferecida no município.

Foram incluídas neste estudo todas as parturientes residentes em área rural ou urbana deste município, cujo parto tenha ocorrido em uma das duas únicas maternidades locais (Hospital Universitário da FURG e Santa Casa de Misericórdia) entre $1^{\circ}$ de janeiro e 31 de dezembro de 2013. Mulheres que tiveram filhos com peso ao nascer inferior a $500 \mathrm{~g}$ e/ou que nasceram antes das 20 semanas de gestação foram consideradas inelegíveis ${ }^{15}$.

O número de parturientes incluídas neste estudo (2.685) é suficiente para avaliar uma prevalência estimada de DL intensa de $25 \%$, a um nível de confiança de 95\%, margem de erro de 2 pontos percentuais e acréscimo de $10 \%$ para eventuais perdas. Para estudo dos fatores associados ao desfecho, houve poder estatístico mínimo de $80 \%$ para examinar a associação do desfecho com as variáveis independentes de interesse, considerando erro alfa de 0,05 , razão expostos/não expostos de 1 para 5 , prevalência de doença nos não expostos de $20 \%$ e razão de riscos de 1,5 .

A coleta das informações ocorreu por meio de questionário padrão pré-codificado aplicado por entrevistadoras previamente treinadas que visitavam diariamente as maternidades e as enfermarias dos serviços de obstetrícia destes dois hospitais. Ao encontrar alguma parturiente proveniente do município de Rio Grande, a entrevistadora a convidava a participar do estudo. Àquelas que aceitaram participar do estudo, foi aplicado o termo de consentimento livre e esclarecido. O questionário buscava informações sobre características demográficas (idade, cor da pele, se vive ou não com companheiro), socioeconômicas (escolaridade, se realiza trabalho remunerado e renda mensal no mês anterior à entrevista), comportamentais (prática de atividade física durante a gestação e tabagismo (nunca fumou, ex-fumante, fumante), nutricionais (índice de massa corporal - IMC no início da gestação) e 
história reprodutiva (paridade, realização de cesarianas prévias e peso ao nascer do filho).

A DL foi caracterizada através da aplicação de uma versão modificada do questionário Nórdico $^{16}$. As parturientes que afirmaram ter tido dor nas costas no último ano eram orientadas a apontar a localização exata da dor em uma figura humana de costas com as regiões cervical, torácica e lombar da coluna vertebral, cada uma delas identificadas por uma cor ${ }^{17,18}$. As parturientes que apontaram para a região lombar, área indicada na cor vermelha, foram consideradas como tendo dor lombar. Se esta dor teve início durante a gestação, ficava estabelecido o diagnóstico de dor lombar gestacional.

Para investigação da intensidade da DL utilizou-se a escala visual analógica para dor, na qual as parturientes atribuíram uma nota entre 0 e 10, considerando 0 "sem dor" e 10 a pior dor possível. Foram considerados casos de DL intensa as parturientes que atribuíram notas entre 7 a 10 para a DLG9.

A análise dos dados foi realizada no pacote estatístico Stata 11. Inicialmente procedeu-se a análise descritiva estratificada segundo o nível de DL para cada variável de interesse utilizando o teste qui-quadrado de heterogeneidade para comparação de proporções. Em seguida, foram realizadas as análises necessárias para calcular a prevalência e examinar os fatores associados à DL intensa entre parturientes com DLG. Foram realizados os cálculos das razões de prevalência (RP) com os respectivos intervalos de confiança de 95\% (IC 95\%) através regressão de Poisson bruta e ajustada com variância robusta. A significância estatística das associações foi avaliada através dos testes Wald heterogeneidade para variáveis dicotômicas/politômicas ou de tendência linear para variáveis ordinais. Foram levadas para a análise ajustada as variáveis independentes que apresentaram valor $\mathrm{p}$ de significância estatística $<0,2$ na análise bruta.

A análise ajustada foi realizada obedecendo a um modelo hierárquico previamente definido com três níveis ${ }^{19}$. No primeiro nível, mais distal, foram incluídas variáveis demográficas da mãe (idade, cor da pele autorreferida, estado civil, escolaridade) e socioeconômica da família (quartil de renda familiar e trabalho durante a gravidez). No segundo, características comportamentais e nutricionais (tabagismo, exercício físico na gestação e IMC antes de engravidar), e no terceiro, variáveis relativas à história obstétrica (paridade, número de cesarianas prévias, peso ao nascer do filho). Mantiveram-se no modelo as variáveis com p-valor $<0,20$, sendo consideradas associadas aquelas com $\mathrm{p}$-valor $<0,05$.

Para avaliar a limitação por DL intensa, foi realizada análise comparativa entre as parturientes com e sem DL intensa, (nota para dor lombar $\geq 7$ e $\leq 6)$ em relação às variáveis "deixar de fazer as lidas da casa", 'faltar ao trabalho" e "procurar por médico".

O projeto Perinatal 2013 foi submetido e aprovado pelo Comitê de Ética em Pesquisa na Área da Saúde da Universidade Federal do Rio Grande (CEPAS/FURG) e pelo CEPAS da Irmandade Santa Casa de Rio Grande. Todas as participantes assinaram um termo de consentimento livre e esclarecido. Foi garantida a confidencialidade das informações prestadas e a possibilidade de deixar o estudo a qualquer momento.

\section{Resultados}

O presente estudo incluiu 2685 parturientes, sendo que metade (50,5\%) tinha entre 20 e 29 anos de idade, $66,2 \%$ eram de cor da pele branca, $85,8 \%$ viviam com companheiro, $44,7 \%$ tinham entre 9 e 11 anos completos de escolaridade e 43,6\% delas trabalharam fora de casa durante a gestação. Cerca de $17 \%$ eram obesas no período pré-gestacional, $23,8 \%$ já haviam passado por pelo menos uma cesariana, 16,3\% eram fumantes e $67,5 \%$ não praticaram atividade física durante a gestação (Tabela 1 ).

As prevalências de DLG e de DL intensa foram respectivamente de 51,2\% e 27,3\%. De acordo com a análise bivariada, a prevalência de DL intensa foi maior nas parturientes com 13-19 anos e naquelas com 5 a 8 anos de escolaridade (Tabela 2). Mulheres com IMC baixo tiveram uma prevalência significativamente menor de DL intensa em relação às eutróficas, com sobrepeso e obesas. Ex-fumantes, assim como mulheres que praticaram atividade física durante a gestação tiveram maior prevalência do desfecho em relação aos seus grupos de comparação (Tabela 2). Não houve diferença estatisticamente significativa nas proporções de parturientes com DL intensa para as variáveis cor da pele, estado civil, renda, trabalho durante a gestação, paridade e número de cesarianas prévias (Tabela 2).

Em relação à análise ajustada, as $\mathrm{RP}$ para mães com 13-19 e 20-29 anos de idade foram respectivamente de 1,23 (IC95\% 1,01-1,50) e 1,22 (IC95\% 1,05-1,41) em relação àquelas com 30 anos ou mais. Mães eutróficas RP 1,69 (IC95\% 1,15-2,50), com sobrepeso RP 1,92 (IC95\% 1,29- 
Tabela 1. Distribuição das parturientes que tiveram filho no município de Rio Grande, RS, em 2013.

\begin{tabular}{|c|c|c|}
\hline Variável & $\mathbf{N}$ & Percentual \\
\hline \multicolumn{3}{|l|}{ Idade materna (anos) } \\
\hline 13-19 & 464 & $17,3 \%$ \\
\hline $20-29$ & 1355 & $50,5 \%$ \\
\hline 30 ou mais & 866 & $32,2 \%$ \\
\hline \multicolumn{3}{|c|}{ Cor da pele (auto-referida) } \\
\hline Branca & 1776 & $66,2 \%$ \\
\hline Parda & 597 & $22,2 \%$ \\
\hline Preta & 312 & $11,6 \%$ \\
\hline \multicolumn{3}{|c|}{ Viviam com companheiro } \\
\hline Não & 382 & $14,2 \%$ \\
\hline Sim & 2303 & $85,8 \%$ \\
\hline \multicolumn{3}{|c|}{ Escolaridade Materna (anos) } \\
\hline $1-4$ & 162 & $6,0 \%$ \\
\hline $5-8$ & 902 & $33,6 \%$ \\
\hline $9-11$ & 1201 & $44,7 \%$ \\
\hline 12 ou mais & 420 & $15,7 \%$ \\
\hline \multicolumn{3}{|c|}{ Quartil de renda familiar } \\
\hline $1^{\circ}($ pior $)$ & 680 & $25,3 \%$ \\
\hline $2^{\circ}$ & 693 & $25,8 \%$ \\
\hline $3^{\circ}$ & 641 & $23,9 \%$ \\
\hline $4^{\circ}$ (melhor) & 671 & $25,0 \%$ \\
\hline \multicolumn{3}{|c|}{ Trabalhou durante a gravidez } \\
\hline Não & 1513 & $56,4 \%$ \\
\hline $\operatorname{Sim}$ & 1172 & $43,6 \%$ \\
\hline \multicolumn{3}{|l|}{ Paridade } \\
\hline Nenhum & 1271 & $47,3 \%$ \\
\hline Um & 795 & $29,6 \%$ \\
\hline Dois ou mais & 619 & $23,1 \%$ \\
\hline
\end{tabular}

\begin{tabular}{|c|c|c|}
\hline Variável & $\mathbf{N}$ & Percentual \\
\hline \multicolumn{3}{|l|}{$\begin{array}{l}\text { Índice de Massa corporal antes } \\
\text { de engravidar (n-2316) }\end{array}$} \\
\hline Baixo & 132 & $5,7 \%$ \\
\hline Normal & 1150 & $49,6 \%$ \\
\hline Sobrepeso & 645 & $27,9 \%$ \\
\hline Obesidade & 389 & $16,8 \%$ \\
\hline \multicolumn{3}{|l|}{ Peso ao nascer do filho $(\mathrm{g})$} \\
\hline Menor 2500 & 260 & $9,7 \%$ \\
\hline $2500-3000$ & 626 & $23,3 \%$ \\
\hline $3000-3999$ & 1654 & $61,6 \%$ \\
\hline Mais de 4000 & 145 & $5,4 \%$ \\
\hline \multicolumn{3}{|l|}{ Ocorrência prévia de cesariana } \\
\hline Não & 2044 & $76,2 \%$ \\
\hline Sim & 639 & $23,8 \%$ \\
\hline \multicolumn{3}{|l|}{ Paridade } \\
\hline Nenhuma & 1271 & $47,3 \%$ \\
\hline Uma & 795 & $29,6 \%$ \\
\hline Duas ou mais & 619 & $23,1 \%$ \\
\hline \multicolumn{3}{|l|}{ Tabagismo } \\
\hline Não & 1925 & $71,7 \%$ \\
\hline Ex-fumante & 321 & $12,0 \%$ \\
\hline Fumante & 439 & $16,3 \%$ \\
\hline Fazia exercício físico na gestação & 873 & $32,5 \%$ \\
\hline $\begin{array}{l}\text { Prevalência de dor lombar } \\
\text { gestacional }\end{array}$ & 1375 & $51,2 \%$ \\
\hline $\begin{array}{l}\text { Prevalência de dor lombar } \\
\text { intensa }\end{array}$ & 732 & $27,3 \%$ \\
\hline
\end{tabular}

2,85) e obesidade RP 2,00 (IC95\% 1,33-3,02) anteriormente à gestação apresentaram maior risco de DL intensa em relação às que tinham baixo peso. Ex-fumantes RP 1,22 (IC95\% 1,02-1,47) e parturientes que praticaram atividade física na gestação RP 1,19 (IC95\% 1,04-1,37) tiveram maior risco para o desfecho em relação aos grupos de comparação. As variáveis escolaridade e peso ao nascer do filho não mantiveram associação com o desfecho na análise ajustada (Tabela 2).

Entre as parturientes que tiveram DL intensa durante a gestação, 29,2\%, 37,3\% e 33,3\% tiveram que deixar de fazer as lidas da casa, faltar ao trabalho e procurar um médico devido à DL, respectivamente. Entre aquelas sem DL intensa, estas proporções foram respectivamente de $9,1 \%$, $11,0 \%$ e 16,1\% (Figura 1).

\section{Discussão}

De acordo com os resultados deste estudo, 51,2\% das entrevistadas tiveram DLG durante a gestação e 27,3\% relataram DL intensa. Parturientes adolescentes, que tinham IMC normal/sobrepeso/obesidade, ex-fumantes e que praticaram atividade física na gestação apresentaram maior risco para DL intensa. Além disso, parturientes com DL intensa tiveram maior limitação para as lidas da casa, maior absenteísmo e maior procura por médico do que os casos de DL não intensa.

A alta prevalência de DL intensa é semelhante à encontrada na literatura ${ }^{8}$ e pode ser considerada um achado preocupante, uma vez que sintomas mais severos resultam em maior limitação na realização das atividades de vida diária e aumentam o risco de cronificação após o parto ${ }^{1}$. 
Tabela 2. Prevalência e fatores associados à dor lombar intensa no período gestacional entre parturientes. Rio Grande, RS, 2013. $(\mathrm{n}=2683)$

\begin{tabular}{|c|c|c|c|c|}
\hline \multirow{2}{*}{ Nível } & \multirow{2}{*}{ Variável } & \multirow{2}{*}{$\begin{array}{c}\text { Prevalência de dor } \\
\text { lombar intensa na gestação }\end{array}$} & \multicolumn{2}{|c|}{ Razão de prevalências e IC95\% } \\
\hline & & & Bruta & Ajustada \\
\hline \multirow[t]{24}{*}{ I } & Idade (anos) & & $<0,002^{\star *}$ & $<0,020^{\star *}$ \\
\hline & 13-19 & $30,2 \%$ & $1,30(1,08-1,56)$ & $1,23(1,01-1,50)$ \\
\hline & $20-29$ & $28,9 \%$ & $1,24(1,07-1,44)$ & $1,22(1,05-1,41)$ \\
\hline & 30 ou mais & $23,2 \%$ & 1,00 & 1,00 \\
\hline & Cor da pele (auto-referida) & & $0,502^{\star * \star}$ & \\
\hline & Branca & $26,7 \%$ & 1,00 & \\
\hline & Parda & $29,2 \%$ & $1,09(0,94-1,26)$ & - \\
\hline & Preta & $27,0 \%$ & $1,01(0,83-1,23)$ & - \\
\hline & Vive com companheiro & & $0,445^{\star * *}$ & \\
\hline & Não & $25,7 \%$ & 1,00 & \\
\hline & Sim & $27,6 \%$ & $1,08(0,89-1,30)$ & \\
\hline & Escolaridade materna (anos) & & $0,036^{\star * \star}$ & 0,160 \\
\hline & $1-4$ & $21,0 \%$ & 1,00 & 1,00 \\
\hline & $5-8$ & $30,2 \%$ & $1,44(1,05-1,97)$ & $1,38(1,00-1,89)$ \\
\hline & $9-11$ & $26,9 \%$ & $1,28(0,94-1,75)$ & $1,26(0,92-1,72)$ \\
\hline & 12 ou mais & $24,5 \%$ & $1,17(0,83-1,65)$ & $1,20(0,85-1,69)$ \\
\hline & Quartil de renda familiar & & $0,440^{\star \star}$ & \\
\hline & $1^{\circ}($ pior $)$ & $25,9 \%$ & 1,00 & \\
\hline & $2^{\circ}$ & $26,6 \%$ & $1,02(0,86-1,22)$ & \\
\hline & $3^{\circ}$ & $30,1 \%$ & $1,16(0,98-1,38)$ & \\
\hline & $4^{\text {o }}$ (melhor) & $26,7 \%$ & $1,03(0,86-1,23)$ & \\
\hline & Trabalhou durante a gravidez & & $0,33^{\star * *}$ & \\
\hline & Não & $28,0 \%$ & 1,00 & \\
\hline & Sim & $26,3 \%$ & $0,94(0,83-1,06)$ & \\
\hline \multirow[t]{12}{*}{ II } & IMC antes de engravidar* & & $0,030^{* *}$ & $0,001^{* *}$ \\
\hline & Baixo & $16,7 \%$ & 1.00 & 1.00 \\
\hline & Normal & $26,8 \%$ & $1,61(1,08-2,38)$ & $1,69(1,15-2,50)$ \\
\hline & Sobrepeso & $29,0 \%$ & $1,74(1,17-2,60)$ & $1,92(1,29-2,85)$ \\
\hline & Obesidade & $28,8 \%$ & $1,73(1,14-2,61)$ & $2,00(1,33-3,02)$ \\
\hline & Tabagismo & & $0,064^{\star * \star}$ & $0,040^{\star * *}$ \\
\hline & Não & $26,9 \%$ & 1,00 & 1,00 \\
\hline & Ex-fumante & $32,4 \%$ & $1,21(1,01-1,44)$ & $1,22(1,02-1,47)$ \\
\hline & Fumante & $25,3 \%$ & $0,94(0,79-1,13)$ & $0,91(0,74-1,11)$ \\
\hline & Exercício físico na gestação & & $0,025^{\star * \star}$ & 0,012 \\
\hline & Não & $26,0 \%$ & 1,00 & 1,00 \\
\hline & Sim & $30,1 \%$ & $1,16(1,02-1,32)$ & $1,19(1,04-1,37)$ \\
\hline \multirow[t]{12}{*}{ III } & Paridade & & $0,240^{* *}$ & \\
\hline & Nenhum & $28,1 \%$ & 1,00 & \\
\hline & Um & $27,5 \%$ & $0,90(0,77-1,06)$ & \\
\hline & Dois ou mais & $25,4 \%$ & $0,98(0,85-1,13)$ & \\
\hline & Número de cesarianas prévias & & $0,660^{\star * *}$ & \\
\hline & Nenhuma & $27,1 \%$ & 1,00 & \\
\hline & Uma ou mais & $27,9 \%$ & $1,03(0,90-1,19)$ & \\
\hline & Peso ao nascer do filho $(\mathrm{g})$ & & $0,020^{\star *}$ & $0,300^{* *}$ \\
\hline & Menor 2500 & $26,5 \%$ & 1,00 & 1,00 \\
\hline & $2500-3000$ & $22,4 \%$ & $0,84(0,66-1,08)$ & $0,78(0,60-1,02)$ \\
\hline & $3000-3999$ & $29,0 \%$ & $1,09(0,88-1,36)$ & $0,97(0,77-1,22)$ \\
\hline & Mais de 4000 & $30,3 \%$ & $1,14(0,83-1,57)$ & $0,99(0,69-1,40)$ \\
\hline
\end{tabular}

*369 indivíduos sem informação ${ }^{* *}$ Teste Wald de tendência linear ${ }^{* * *}$ Teste Wald de heterogeneidade. 


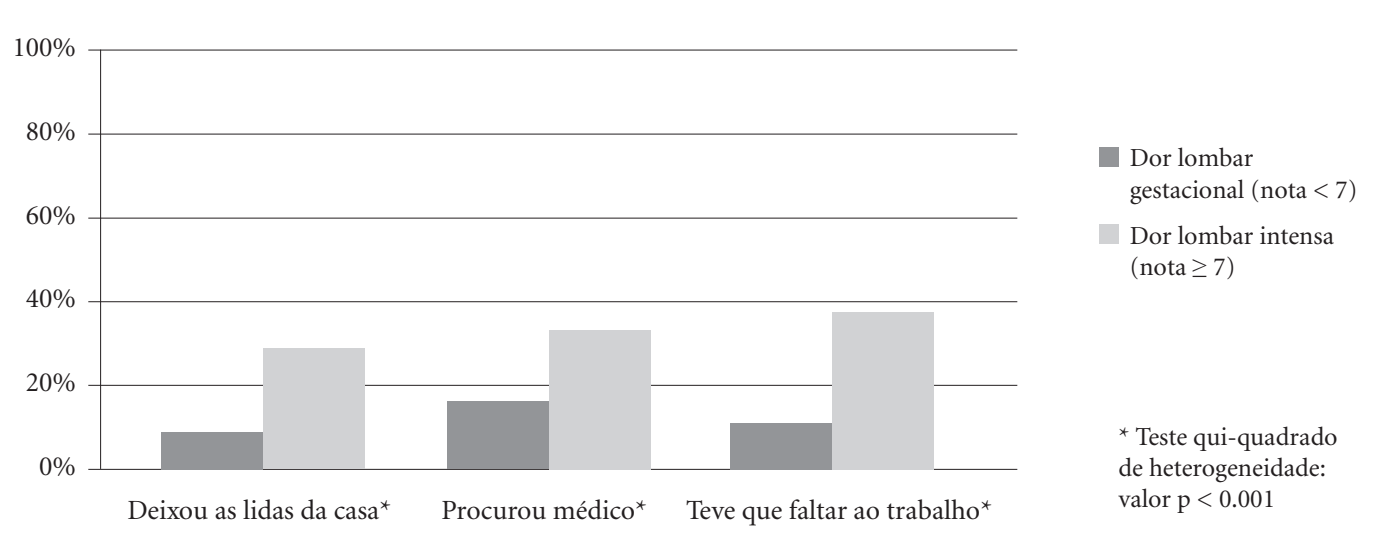

Figura 1. Limitações e procura por atendimento médico em gestantes de acordo com a intensidade dos sintomas: dor lombar gestacional (nota $<$ 7) e dor lombar intensa (nota $\geq 7$ ). Rio Grande, RS, 2013.

Uma proporção significativa das mulheres com sintomas mais severos deixou de fazer as lidas da casa e faltou ao trabalho, concordando com a literatura que mostra que $8 \%$ das gestantes com DL fica com alguma incapacidade ${ }^{2,8,20}$. Entretanto, apenas um terço das mulheres com DL intensa procurou um médico, o que pode ser decorrente do fato de que tanto gestantes quanto profissionais de saúde tendem a minimizar os sintomas e as limitações por considerarem a DL normal para o período ${ }^{3}$. Além disso, a literatura relata que existe falta de conhecimento sobre quais são as formas eficazes de tratamento que não impliquem em ameaça à gestante e ao desenvolvimento do feto ${ }^{2}$.

$\mathrm{O}$ maior risco de DL intensa em adolescentes e mulheres entre 20-29 anos tem consistência com um dos estudos identificados, embora este não tenha avaliado a severidade da dor $^{7}$. A sobrecarga na coluna lombar, juntamente com as alterações fisiológicas das estruturas musculoesqueléticas da região lombo pélvica, pode ter maior impacto nas gestantes de menor idade, especialmente nas adolescentes, que ainda estão em fase de crescimento. Embora a associação entre menor idade e DL gestacional seja controversa por serem mais comuns resultados apontando para maior prevalência entre gestantes de maior idade $^{1}$, a maioria dos estudos não avaliam a gravidade dos sintomas.

$\mathrm{O}$ aumento do risco de DL intensa na medida em que aumenta o IMC pré-gestacional pode ter relação com o fato de que sobrepeso/obesidade são associados à DL na população em geral ${ }^{21}$. De acordo com a literatura, o aumento do peso corporal resulta em maior sobrecarga às estruturas ósteo-músculo-ligamentares da região lombar, predispondo ao aparecimento de sintomas no período pré-gestacional. Na gestação, estes sintomas podem ser agravados devido ao aumento ainda maior dessa sobrecarga em decorrência das alterações fisiológicas da região lombo-pélvica. Além disso, existem evidências de que mulheres com DL prévia têm maior risco de desenvolver $\mathrm{DLG}^{2}$.

$\mathrm{Na}$ população geral, fumantes e ex-fumantes tem maior prevalência de DL em relação a não fumantes ${ }^{22}$, embora esta associação não seja unânime em gestantes. ${ }^{4}$ Neste estudo foi avaliado um desfecho de maior severidade, o que não ocorre na maioria dos estudos sobre DLG, de forma que os efeitos do tabagismo em ex-fumantes podem ter resultado em dores mais intensas devido à ação da nicotina sobre os receptores algógenos do cérebro ${ }^{22}$. Entretanto, a ausência de efeito sobre gestantes fumantes limita esta inferência e sugere a necessidade de avaliar com mais detalhes os efeitos do tabagismo sobre a DLG.

O maior risco de DL intensa entre as mulheres que fizeram exercício físico na gestação contraria alguns relatos de que gestantes submetidas à atividade física têm menos DL ou menos incapacidade devido à $\mathrm{DLG}^{5,23,24}$. Entretanto, um estudo de meta-análise mostra que há pouca evidência de que exercícios na gestação previnam a DLG, bem como reduzam os níveis de dor ${ }^{25}$. Além disso, o delineamento transversal não permite estabelecer a relação temporal entre a exposição e o desfecho. Isso ocorre porque gestantes com DL 
intensa podem ter sido orientadas a praticar atividade física na busca pelo alívio dos sintomas ${ }^{26}$, modificando assim seu status de exposição em decorrência do desfecho, resultando em causalidade reversa ${ }^{26}$.

Uma limitação que pode ter afetado este estudo diz respeito à definição do desfecho e a não diferenciação entre DL, dor na cintura pélvica e dor na sínfise púbica. Estas dores se sobrepõem no período gestacional, o que pode ter resultado em erro de classificação para algumas parturientes, superestimando a ocorrência do desfecho. O uso da ilustração de uma figura humana mostrando as diversas regiões das costas, dentre as quais a região lombar, está de acordo com um consenso que busca padronizar os estudos sobre dor lombar em nível populacional, e que a caracteriza como aquela dor que se inicia abaixo da $12^{\text {a }}$ costela e se estende até a prega glútea inferior ${ }^{27}$. No entanto, para estudo da DL em gestantes, é preciso melhorar a caracterização do desfecho de forma a discernir apropriadamente dois desfechos distintos, ou seja, a DL gestacional e a dor da cintura pélvica ${ }^{8}$.

Este estudo avaliou a prevalência e os fatores associados à DL gestacional intensa em 2013 no município de Rio Grande. Nenhum outro estudo com igual representatividade, abrangência e baixo número de perdas - incluiu $97 \%$ de todas as gentes de um município de porte médio - foi encontrado para esta população alvo no país.

Desta forma, os resultados aqui apresentados são relevantes por mostrarem que a DL intensa tem alta prevalência e traz limitações importantes às atividades de vida diária das gestantes, bem como identifica as mulheres mais vulneráveis a esta condição. Isso evidencia a necessidade de que os profissionais de saúde estejam atentos à presença de sintomas musculoesqueléticos na gestação, sobretudo, a dor lombar, e que devem considerar a abordagem deste problema durante as consultas de rotina do pré-natal.

\section{Colaboradores}

VM Duarte e RD Meucci realizaram a revisão de literatura, a análise dos dados e elaboração do artigo. JA Cesar elaborou o projeto de onde o estudo foi originado, supervisionou a coleta e entrada de dados e revisou o artigo final. Todos os autores contribuíram de maneira fundamental para a realização desse estudo e leram e aprovaram o conteúdo do manuscrito. 


\section{Referências}

1. Borg-Stein J, Dugan SA, Gruber J. Musculoskeletal aspects of pregnancy. Am J Phys Med Rehabil 2005; 84(3):180-192.

2. Vermani FE, Mittal FR, Weeks MA. Pelvic Girdle Pain and Low Back Pain in Pregnancy: A Review. Pain Practice 2009; 10(1):60-71.

3. Heckman JD, Sassard R. Musculoskeletal considerations in pregnancy. J Bone Joint Surg Am 1994; 76(11):1720-1730.

4. Vleeming A, Albert HB, Ostgaard HC, Sturesson B, Stuge B. European guidelines for the diagnosis and treatment of pelvic girdle pain. Eur Spine J 2008; 17(6):794-819.

5. Gjestland K, Bø K, Owe KM, Eberhard-Gran M. Do pregnant women follow exercise guidelines? Prevalence data among 3482 women, and prediction of low-back pain, pelvic girdle pain and depression. Br J Sports Med 2013; 47(8):515-520.

6. Kovacs FM, Garcia E, Royuela A, Gonzalez L, Abraira V. Prevalence and factors associated with low back pain and pelvic girdle pain during pregnancy: a multicenter study conducted in the Spanish National Health Service. Spine (Phila Pa 1976) 2012; 37(17):1516-1533.

7. Wang SM, Dezinno P, Maranets I, Berman MR, Caldwell-Andrews AA, Kain ZN. Low back pain during pregnancy: prevalence, risk factors, and outcomes. $\mathrm{Ob}$ stet Gynecol 2004; 104(1):65-70.

8. Wu WH, Meijer OG, Uegaki K, Mens JMA, Diee"n JHv, Wuisman PIJM, Ostgaard HC. Pregnancy-related pelvic girdle pain (PPP), I: Terminology, clinical presentation, and prevalence. Eur Spine Journal 2004; 13(7):575-589.

9. Bergström C, Persson M, Mogren I. Pregnancy-related low back pain and pelvic girdle pain approximately 14 months after pregnancy - pain status, self-rated health and family situation. BMC Pregnancy Childbirth 2014; 14:48.

10. Mogren IM, Pohjanen AI. Low back pain and pelvic pain during pregnancy: prevalence and risk factors. Spine (Phila Pa 1976) 2005; 30(8):983-991.

11. Rodrigues WFG, Silva LR, Nascimento MAL, Pernambuco CS, Giani TS, Dantas EHM. Prevalência de lombalgias e inatividade física: o impacto dos fatores psicossociais em gestantes atendidas pela Estratégia de Saúde da Família. Einstein (São Paulo) 2011; 9(4):489493.

12. Santos MM, Gallo AP. Lombalgia gestacional: prevalência e características de um programa pré-natal. Arq bras ciênc saúde 2010; 35(3):174-179.

13. Madeira HG, Garcia JB, Lima MV, Serra HO. Disability and factors associated with gestational low back pain. Rev Bras Ginecol Obstet 2013; 35(12):541-548.

14. Mohseni-Bandpei MA, Fakhri M, Ahmad-Shirvani M, Bagheri-Nessami M, Khalilian AR, Shayesteh-Azar M, Mohseni-Bandpei H. Low back pain in 1,100 Iranian pregnant women: prevalence and risk factors. Spine $J$ 2009; 9(10):795-801.

15. Dias-Damé JL, Cesar JA. Disparities in Prevalence of Smoking and Smoking Cessation during Pregnancy: A Population-Based Study. BioMed Res Int 2015; 2015:345430.
16. Kuorinka I, Jonsson B, Kilbom A, Vinterberg H, Biering-Sørensen F, Andersson G, et al. Standardised Nordic questionnaires for the analysis of musculoskeletal symptoms. Appl Ergon 1987; 18(3):233-237.

17. Meucci RD, Fassa AG, Paniz VM, Silva MC, Wegman $\mathrm{DH}$. Increase of chronic low back pain prevalence in a medium-sized city of southern Brazil. BMC Musculoskelet Disord 2013; 14:155.

18. Silva MC, Fassa AG, Valle NC. Chronic low back pain in a Southern Brazilian adult population: prevalence and associated factors. Cad Saude Publica 2004; 20(2):377385.

19. Victora CG, Huttly SR, Fuchs SC, Olinto MTA. The role of conceptual frameworks in epidemiological analysis: a hierarchical approach. Int J Epidemiol 1997; 26(1):224-227.

20. Gutke A, Olsson CB, Völlestad N, Öberg B, Wikmar LN, Robinson HS. Association between lumbopelvic pain, disability and sick leave during pregnancy - a comparison of three Scandinavian cohorts. J Rehabil Med 2014; 46(5):468-474

21. Shiri R, Karppinen J, Leino-Arjas P, Solovieva S, Viikari-Juntura E. The association between obesity and low back pain: a meta-analysis. Am J Epidemiol 2010; 171(2):135-154.

22. Shiri R, Karppinen J, Leino-Arjas P, Solovieva S, Viikari-Juntura E. The association between smoking and low back pain: a meta-analysis. Am J Med 2010; 123(1):87.e7-.e35

23. Ostgaard HC, Zetherstrom G, Roos-Hansson E, B BS. Reduction of back and posterior pelvic pain in pregnancy. Spine (Phila Pa 1976). 1994; 19(8):894-900.

24. Stafne SN, Salvesen KA, Romundstad PR, Britt S, Morkved S. Does regular exercise during pregnancy influence lumbopelvic pain? A randomized controlled trial. Acta Obstet Gynecol Scand 2012; 91(5):552-559.

25. Liddle SD, Pennick V. Interventions for preventing and treating low-back and pelvic pain during pregnancy. Cochrane Database Syst Rev 2015; (9):CD001139.

26. Gordis L. Epidemiology. $4^{\text {th }}$ ed. Philadelphia: Saunders Elsevier; 2009.

27. Dionne CE, Dunn KM, Croft PR, Alf L. Nachemson, Buchbinder R, Walker BF, Wyatt M, Cassidy JD, Rossignol M, Leboeuf-Yde C, Hartvigsen J, Leino-Arjas P, Latza U, Reis S, Gil Del Real MT, Kovacs FM, Oberg B, Cedraschi C, Bouter LM, Koes BW, Picavet HS, van Tulder MW, Burton K, Foster NE, Macfarlane GJ, Thomas E, Underwood M, Waddell G, Shekelle P, Volinn E, Von Korff M. A Consensus Approach Toward the Standardization of Back Pain Definitions for Use in Prevalence Studies. Spine (Phila Pa 1976) 2008; 33(1):95-103.

Artigo apresentado em 23/05/2016

Aprovado em 21/08/2016

Versão final apresentada em 23/08/2016 